of this association to facilitate rapid diagnosis and management of AIN.

Original article Geevasinga $\mathrm{N}$ et al. (2006) Proton pump inhibitors and acute interstitial nephritis. Clin Gastroenterol Hepatol 4: 597-604

\section{Could MELD be improved by incorporating serum sodium level?}

The Model for End-stage Liver Disease (MELD) score-derived from serum total bilirubin and creatinine levels, plus the international normalized ratio-is commonly used in the US to assess patients' medical urgency for liver transplantation. Previous studies indicated that hyponatremia is also an important independent predictor of mortality in patients awaiting liver transplant, and Biggins et al. have confirmed these findings. These authors have developed a novel 'MELD-Na' score, which incorporates hyponatremia (i.e. serum sodium levels in the range $120-135 \mathrm{mmol} / \mathrm{l}$ ) into the standard MELD. They recommend that data collected by the United Network for Organ Sharing in the US should be analyzed, to validate their modified MELD.

This prospective study was specifically designed to evaluate and optimize the MELD score in relation to predicting transplant waitinglist mortality. The patient cohort comprised 753 predominantly white patients awaiting liver transplant under the current US MELD-based organ-allocation system, for whom complete records were available. In this cohort, use of the optimized MELD-Na would have changed graft allocation in up to $27 \%$ of cases.

The authors note that the contribution of hyponatremia to risk of death is probably less important in patients with a very high MELD score, who are already at high risk of death. They call for studies involving large numbers of such patients, to evaluate the interaction between hyponatremia and a high MELD score, and to refine the MELD-Na model further.

Original article Biggins SW et al. (2006) Evidence-based incorporation of serum sodium concentration into MELD. Gastroenterology 130: 1652-1660

\section{Genetic profiling could identify patients at risk of relapse of colorectal cancer}

Patients who present with early-stage colorectal cancer without metastasis (Dukes' stage A or B) typically undergo surgery without adjuvant therapy. Despite having apparently lowrisk disease, $10-40 \%$ of these patients relapse after surgery, and up to $50 \%$ die from complications or metastasis. Molecular markers that can predict relapse in patients with early-stage colorectal cancer are being investigated, but previous studies analyzed only a few candidate markers.

Al-Mulla and colleagues' study enrolled 70 patients with early-stage, nonmetastatic colorectal cancer. Tumor samples from 27 patients who relapsed after surgery were compared with samples from 43 patients who did not relapse during follow-up (range 2-9 years). The authors used comparative genomic hybridization to identify chromosomal aberrations in a total of 61 tumors; their findings were confirmed by DNA microarray analysis of 10 tumors.

This genome-wide analysis identified many abnormalities that correlated with relapse of early-stage colorectal cancer after surgery, including loss of chromosomes $1 p, 4,5 q$, $8 p, 9 p, 14 q$, and $18 p$, and gain of chromosomes 1q, 8q, and 13q. Notably, samples from patients who relapsed were more likely to have multiple aberrations (e.g. loss of both $8 p$ and 18q, and loss of both 4 and 14q). These specific multiple aberrations were associated with markedly short survival after surgery. Loss of chromosome 4 independently predicted poor survival.

The same team has recently used an unrelated technique (analysis of highly polymorphic microsatellite markers) to narrow down the deletions on chromosomes 4 and 14 to specific regions.

Original articles Al-Mulla F et al. (2006) Genetic profiling of stage I and II colorectal cancer may predict metastatic relapse. Mod Pathol 19: 648-658

Al-Mulla F et al. (2006) Metastatic recurrence of early-stage colorectal cancer is linked to loss of heterozygosity on chromosomes 4 and 14q. J Clin Pathol 59: 624-630 